\title{
Raman and infrared study of the quasi-one-dimensional betaine arsenate-phosphate mixed-crystal system
}

\author{
S. Lanceros-Méndez \\ Departamento de Física, Universidade do Minho, 4710-057 Braga, Portugal \\ H. Ebert and G. Schaack \\ Physikalisches Institut der Universität Würzburg, Am Hubland, D-97074 Würzburg, Germany \\ A. Klöperpieper \\ Fachbereich Physik der Universität des Saarlandes, D-66123 Saarbrücken, Germany
}

(Received 17 July 2002; published 28 January 2003)

\begin{abstract}
Ferroelectric (FE) betaine arsenate (BA) and antiferroelectric (AF) betaine phosphate (BP) are structurally very closely related and mixed crystals $\mathrm{BA}_{x} \mathrm{BP}_{1-x}$ can be grown over the full concentration range. This system exhibits interesting pressure-temperature-concentration phase diagrams in which the transition in concentration from an AF to a FE phase can be observed with a concentration-dependent appearance of some mixed regions. At low temperatures different relaxation processes have been observed that might be related to the transition from a domain state to an orientational glass depending on concentration. In some regions of the phase diagram different phases coexist. The origin of the different transitions and relaxation processes is still unclear and recent $\mathrm{x}$-ray-diffraction studies reveal that the peculiar behavior of the mixed crystals cannot be explained from the structural point of view. We have performed Raman and infrared investigation of samples across the whole $(x, T)$ phase diagram, at various hydrostatic pressures, in order to shed light on the structural origin of the different processes. It is concluded that the FE or AF character of the ordered phase is related to the degrees of freedom of the $\mathrm{AsO}_{4}$ and $\mathrm{PO}_{4}$ tetrahedra. In the same way these units determine the type and the modes triggering the phase transitions for each concentration. The latter seems to occur together with a participation of the betaine molecule, especially of the methyl and buthyl groups.

DOI: 10.1103/PhysRevB.67.014109

PACS number(s): 77.80.-e, 72.80.Ng, 78.30.-j, 87.64.Je
\end{abstract}

\section{INTRODUCTION}

Ferroelectric (FE) betaine arsenate (BA) $\left[\left(\mathrm{CH}_{3}\right)_{3} \mathrm{NCH}_{2} \mathrm{COO} \cdot \mathrm{H}_{3} \mathrm{AsO}_{4}\right]$, antiferroelectric (AF) betaine phosphate (BP) $\left[\left(\mathrm{CH}_{3}\right)_{3} \mathrm{NCH}_{2} \mathrm{COO} \cdot \mathrm{H}_{3} \mathrm{PO}_{4}\right]$, and $\mathrm{FE}$ betaine phosphite (BPI) $\left[\left(\mathrm{CH}_{3}\right)_{3} \mathrm{NCH}_{2} \mathrm{COO} \cdot \mathrm{H}_{3} \mathrm{PO}_{3}\right]$ are structurally very closely related and mixed crystals are formed accordingly: $\mathrm{BA}_{x} \mathrm{BP}_{1-x}, \mathrm{BP}_{x} \mathrm{BPI}_{1-x}, \quad 0 \leqslant x \leqslant 1$. Glassy phases attributed to frustrated $\mathrm{FE}$ and $\mathrm{AF}$ interactions, and proton glass behavior, has been proposed in some mixed crystals of these families $\left[\mathrm{BA}_{x} \mathrm{BP}_{1-x}\right.$ (Refs. 1-10) and $\mathrm{BP}_{x} \mathrm{BPI}_{1-x}$ (Refs. 11-13). Though the phase diagram of the $\mathrm{BP}_{x} \mathrm{BPI}_{1-x}$ mixed-crystal system shows the typical features of the dipole glasses, such as the $\mathrm{Rb}_{x}\left(\mathrm{ND}_{4}\right)_{1-x} \mathrm{D}_{2} \mathrm{PO}_{4}$ (Ref. 3) mixed system, no convincing evidence of such behavior has been found in the $\mathrm{BA}_{x} \mathrm{BP}_{1-x}$ system. Further, a rather complex phase diagram with different mixed phase regions, transitions, and relaxations have been found and deserves deeper investigation (Fig. 1).

The crystal structures, of both BP (Ref. 14) and BA, ${ }^{15}$ display as a common feature the existence of quasi-onedimensional (quasi-1D) chains of arsenate or phosphate tetrahedra, interconnected by hydrogen bonds. One betaine molecule is attached to each tetrahedron by two $\mathrm{H}$ bonds via the carboxyl groups, which differ in strength in the case of BP but have approximately equal strength in BA. As a consequence, the deformations of the tetrahedra are subtly different in both compounds, resulting in their slightly different steric behavior. The length $d_{\mathrm{H}}$ of the hydrogen bonds interconnecting the tetrahedra, on the other hand, are almost identical in both compounds $\left(2.485 \leqslant d_{\mathrm{H}} \leqslant 2.533 \AA\right)$. The bond length $d_{\mathrm{H}}$ lies within the narrow region, where doubleminimum potentials are to be expected for $\mathrm{H}$ bonds. The single chains in both BA and BP are ferroelectrically ordered; the interchain interaction, however, slightly differs in both crystals, resulting in the FE order of $\mathrm{BA}$ and the $\mathrm{AF}$ order in BP.

On the other hand, BP and BA are not isostructural with each other. The space group of BP is $P 2_{1} / m(Z=2)$ above $365 \mathrm{~K}, P 2{ }_{1} / c(Z=4)$ in phase II between 365 and $86 \mathrm{~K}$, and $P 2{ }_{1} / c(Z=8)$ at lower temperature. ${ }^{14}$ In phase I, the betaine and phosphate molecules are disordered against the mirror planes perpendicular to the $b$ axis. The molecules are ordered alternatively in phase II with doubling of the cell dimension along the $c$ axis. Below $86 \mathrm{~K}$, the unit cell is further elongated twice along the $a$ axis. The disordered hydrogen atoms between the phosphate tetrahedra become ordered in the lowtemperature phase accompanied by small antiferroelectric displacements. ${ }^{16}$

The space groups of BA are Pcnm $(Z=4)$ in phase I above $411 \mathrm{~K}, P 2_{1} / n(Z=4)$ in phase II between 411 and $119 \mathrm{~K}$, and $P c(Z=4)$ in phase III, below $119 \mathrm{~K}^{15,17} \mathrm{Al}$ though the local configurations between betaine and arsenate in BA are similar to those between betaine and phosphate in $\mathrm{BP}$, the unit-cell structure is a little different between BA and BP. 


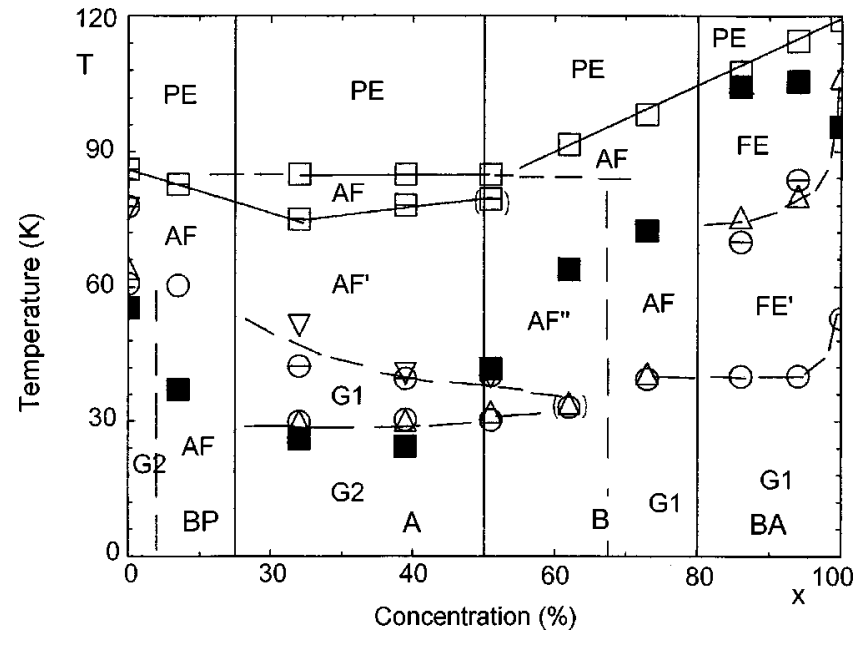

FIG. 1. Transition temperature versus concentration diagram of $\mathrm{BA}_{x} \mathrm{BP}_{1-x}$ for the whole concentration region at $n=100 \mathrm{kHz}$, determined from dielectric measurements (Refs. 1-3). Open squares: anomalies in $\varepsilon^{\prime}(T)$; circles: anomalies in $\tan \delta(T)$; triangles: anomalies in $\varepsilon^{\prime \prime}(T)$. The black squares correspond to the fitted $\mathrm{Cu}-$ rie temperature. The brackets in $\tan \delta(T)$ indicate anomalies much smaller than the others so that we could not observe the corresponding anomaly in $\varepsilon^{\prime \prime}(T)$. The brackets in $\varepsilon^{\prime}(T)$ indicate a small shoulder more than a well-defined anomaly. Solid lines are drawn to guide the eye. The various types of order are indicated. AF: antiferroelectric; $\mathrm{AF}^{\prime}$ : coexistence of $\mathrm{FE}$ (ferroelectric) and $\mathrm{AF}$ components; $\mathrm{AF}^{\prime \prime}$ : coexistence of two AF components; G: Glass phases, G1: Vogel-Fulcher, i.e., collective relaxation and G2: Arrhenius, i.e., single-ion relaxation; and $\mathrm{FE}^{\prime}$ :FE with a domain freezing process. Solid lines are drawn to guide the eyes.

Mixed crystals have been studied previously by Maeda et $\mathrm{al}^{5-7}$ and by Kim et al. $^{8}$ Maeda reported a glasslike behavior for $0.5 \leqslant x \leqslant 0.85$, and Kim observed a dimensionality crossover from one to three dimensions at low temperatures. We have complemented these previous measurements by extending the measurements to lower temperatures and have in addition used hydrostatic pressure and external bias fields. ${ }^{1-4}$ We have studied this system also by measurements of the dielectric hysteresis loops and the current curves (charging and discharging the sample capacitor). ${ }^{4}$ An enriched picture of the system has been obtained ${ }^{1-4}$ (Fig. 1). The concentration-temperature diagram $[(x, T)$ diagram $]$ was divided into the following four different regions (uncertainty: $\approx \pm 5 \%$ for the BP-A and B-BA limits, and $\approx \pm 2 \%$ for the A-B limit) as indicated by the anomalies in $\varepsilon^{\prime}(T)$ and $\tan \delta(T)$ [i.e., $\varepsilon^{\prime \prime}(T)$ ] (Fig. 1):

(i) $\mathrm{BP}$ region: the region occurs up to a $\mathrm{BA}$ concentration of $x \approx 15 \%$; the behavior is similar to that in pure BP, i.e., a phase transition at $T_{c 2}=86 \mathrm{~K}(x=0)$ into an AF phase and another anomaly at $T_{d} \approx 60 \mathrm{~K}(x=0)$ in $\tan \delta(T)$ are encountered, except for the stabilization of the AF phase due to random fields caused by the impurities (which suppress the low-temperature process).

(ii) Intermediate region $\mathrm{A}$ : the concentration region $15 \%$ $\approx x_{1}<x<x_{2} \approx 50 \%$ is characterized by two anomalies in $\varepsilon^{\prime}(T)$ and $\tan \delta(T)$ [i.e., $\left.\varepsilon^{\prime \prime}(T)\right]$.

(iii) Intermediate region $\mathrm{B}$ : in the concentration region
$50 \% \approx x_{2}<x<x_{3} \approx 80 \%$ the double peak structure in $\varepsilon^{\prime}(T)$ disappears in favor of a single, relatively broad anomaly. The same occurs in the behavior of $\tan \delta(T)$ at slightly higher concentrations. Its value decreases very strongly at intermediate concentrations in this concentration region.

(iv) BA region: above a BA concentration of $x \approx 80 \%$ there is no longer a transition into an AF-type phase but into a FE one, as demonstrated by measurements of the dielectric hysteresis. The behavior in $\varepsilon^{\prime}(T), \varepsilon^{\prime \prime}(T)$, and $\tan \delta(T)$ is similar to that in BA but there is an effect of the random fields.

The separation of the $(x, T)$ diagram into these four regions is also supported by the different behavior of the fitted Curie temperature (decreasing, stable, increasing, and decreasing) in each of these regions (Fig. 1).

As a summary, the $\mathrm{BA}_{x} \mathrm{BP}_{1-x}$ system exhibits a $(x, T)$ phase diagram in which the transition in concentration from an AF to a FE phase can be observed with a concentrationdependent appearance of some mixed regions. At low temperatures several relaxations have been observed that might be related to the transition from a domain state to an orientational glass depending on concentration. In some regions of the phase diagram different phases coexist. The origin of the different transitions and relaxation processes it is still unclear and recent $\mathrm{x}$-ray-diffraction studies reveal that the peculiar behavior of the mixed crystals cannot be explained from the structural point of view., ${ }^{9,10}$

Preliminary results on the concentration dependence of the Raman spectra for some mixed crystals were presented in Refs. 1 and 2. In the present paper detailed Raman and infrared measurements at various hydrostatic pressures along the $(x, T)$ phase diagram are presented. Both the temperature dependence of the spectra taken in the different phases and the concentration dependence give insight into the structural origin of this complex phase diagram.

\section{EXPERIMENT}

The crystals were grown by controlled evaporation from aqueous solutions, and their concentrations $x$ subsequently analyzed by means of UV spectroscopy and density methods to an accuracy of about 3\%. For the Raman experiments, oriented samples in the shape of cubes (approximately 8 $\times 5 \times 5 \mathrm{~mm}^{3}$ ) were optically polished; the spectra were excited with the linearly polarized $\lambda=514.5-\mathrm{nm}$ line of an argon-ion laser with a power of about $130 \mathrm{~mW}$ at the entrance of a ${ }^{4} \mathrm{He}$-evaporation cryostat providing the required sample temperature by controlled evaporation and sample heating. Once the temperature was stabilized it could be maintained constant with a variation of $\pm 0.02 \mathrm{~K}$ in each measurement, and $\pm 0.1 \mathrm{~K}$ in $12 \mathrm{~h}$. The light was scattered through $90^{\circ}$ and analyzed with a Dilor XY triple-path spectrometer $(f=50 \mathrm{~cm})$ for the accessible types of symmetries (Table I) in each phase of the phase diagram, between 15 and $3150 \mathrm{~cm}^{-1}$. The uncertainty in the orientation of the sample was $<3^{\circ}$. The instrumental bandwidth was $1 \mathrm{~cm}^{-1}$. The measured concentrations were $x=0.00,0.07,0.24,0.39,0.51$, $0.62,0.73,0.86,0.94$, and 1.00 .

Experiments under hydrostatic pressure were performed in a home-built cryostat and high-pressure chamber. ${ }^{4}$ Helium 
TABLE I. Number of modes, symmetry, and activity for BP and BA in the different phases covered by this investigation. In the remainder of the work the symmetries will be referred to those in the paraelectric phase. Infrared in table denoted by IR.

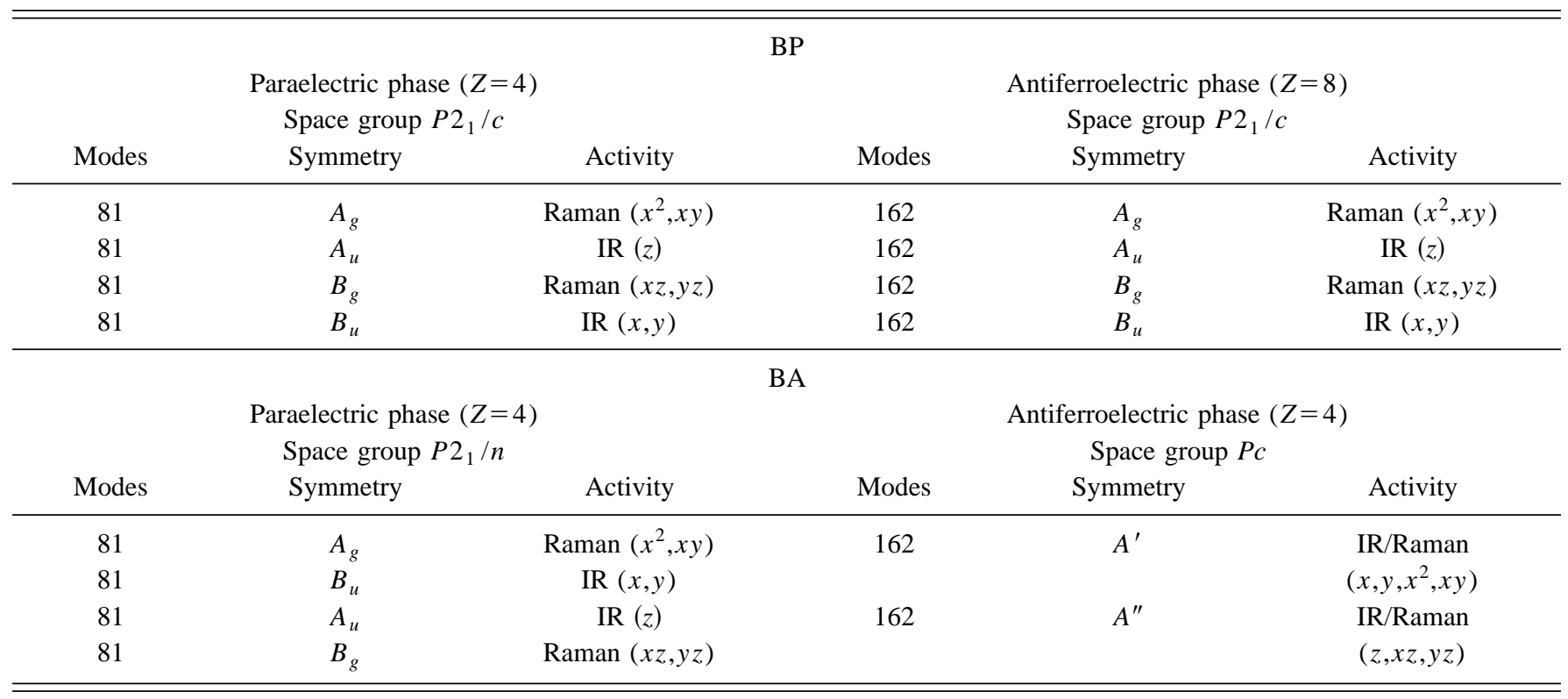

was used as pressure-transmitting medium. The pressure could be changed between normal pressure and $500 \mathrm{MPa}$ with an absolute uncertainty of $\pm 7 \mathrm{MPa}$.

For the far-infrared reflection spectroscopy the samples in the shape of thin slices (approximately $6.5 \times 6.5 \times 1.6 \mathrm{~mm}^{3}$ ) were cut parallel to the monoclinic axis and optically polished. Two spectra in each different phase were taken using a Grubb-Parson Fourier spectrometer for the accessible symmetries (Table I) between 15 and $600 \mathrm{~cm}^{-1}$. The investigated samples were $x=0.00,0.51,0.62$, and 1.00 .

The observed spectra were fitted with a sum over independent damped oscillators: ${ }^{18}$

$$
\varepsilon(\omega)=\varepsilon_{\infty}+\sum_{i} \frac{\Delta \varepsilon_{i} \nu_{i}^{2}}{\nu_{i}^{2}-\nu^{2}+i \nu \gamma_{i}}
$$

This was done in order to observe those modes with anomalies in their energy $\left(\nu_{i}\right)$, damping $\left(\gamma_{i}\right)$, and dielectric strength $\left(\Delta \varepsilon_{i}\right)$ at the different phase transitions.

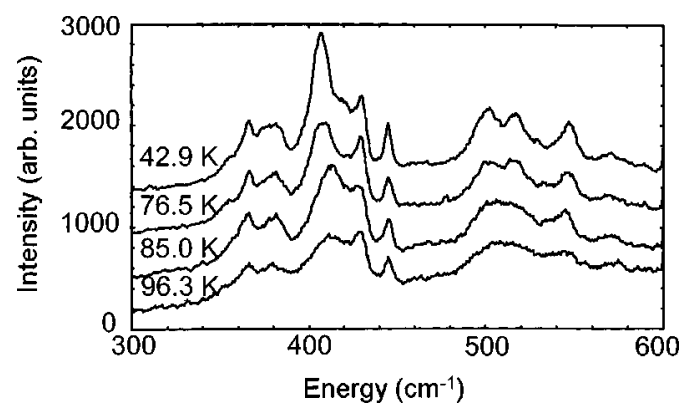

FIG. 2. Raman spectra in $A_{g}$ symmetry [ $\left.X(Y Y) Z\right]$ between 300 and $600 \mathrm{~cm}^{-1}$ in the different phases for pure BP.

\section{EXPERIMENTAL RESULTS}

\section{A. Temperature dependence}

Raman and infrared spectra for each sample were taken at different temperatures and hydrostatic pressures covering the different regions of the phase diagram.

\section{BP region}

Our Raman and infrared spectra for pure BP are consistent with those published in Refs. 19-21, i.e., no soft mode could be detected but new lines appear at 19.2 and $394 \mathrm{~cm}^{-1}$ in $B_{g}$ and at $18.6 \mathrm{~cm}^{-1}$ in $A_{g}$ symmetry, and other lines change abruptly below the transition at $81 \mathrm{~K}$ where a doubling of the unit cell occurs. In the other transitions only small structural changes occur.

The splitting, change in shape, and/or increase of some modes are especially significant in $A_{g}$ symmetry in the regions around 405, 507, and $546 \mathrm{~cm}^{-1}$ (Fig. 2), and in $B_{g}$ symmetry in the region around $2995 \mathrm{~cm}^{-1}$ (Fig. 3). The changes in $A_{g}$ symmetry occur down to the lowest temperature. The spectra taken under pressure present no major differences with respect to those taken at normal pressure. The spectral changes observed under pressure point out that the
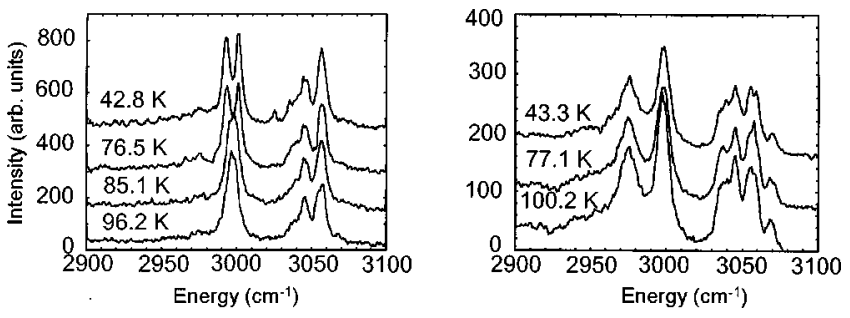

FIG. 3. Comparison of the phase behavior of the mode group around $2995 \mathrm{~cm}^{-1}$ in $B_{g}$ symmetry $[X(Y X) Z]$ in both $\mathrm{BA}_{0.00} \mathrm{BP}_{1.00}$ (left) and $\mathrm{BA}_{0.07} \mathrm{BP}_{0.93}$ (right). 

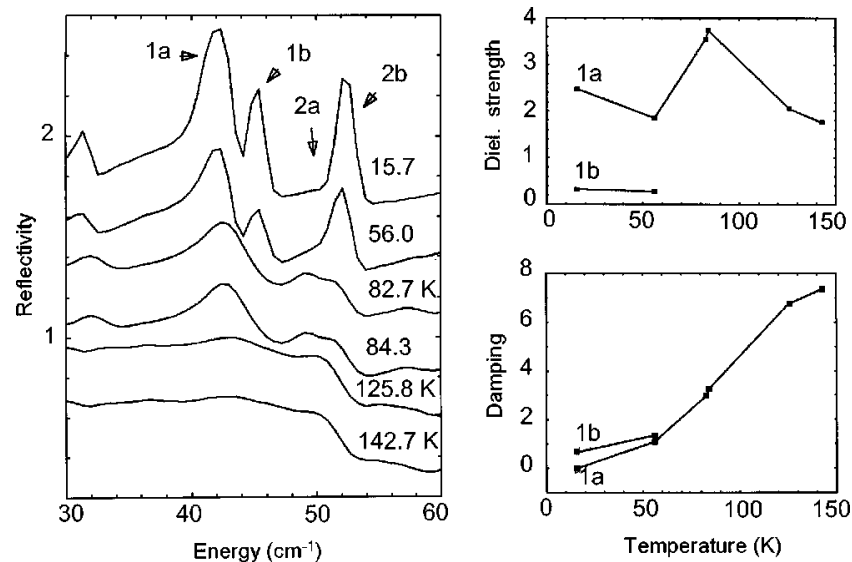

FIG. 4. Typical IR spectra of BP (left) and fitted modes where indications that the three regions of the phase diagram can be observed (right, $A_{u}$ symmetry). Solid lines are drawn to guide the eyes.

transitions at $T_{c 2}$ and $T_{c 3}$ must occur together.

In the IR-reflection spectra 23 and 21 modes were fitted with temperature-dependent frequencies in $A_{u}$ and $B_{u}$ symmetries, respectively. Especially at low wave numbers $(<60$ $\mathrm{cm}^{-1}$, significant indications to three different phases appear, indicated through energy shifts of some modes and anomalies in $\gamma_{i}$ and $\Delta \varepsilon_{i}$.

In $A_{u}$ symmetry a new mode at $44.5 \mathrm{~cm}^{-1}$ appears with cell doubling, see Figs. 4 and 5. The same occurs in the region between 76 and $79 \mathrm{~cm}^{-1}$ in $B_{u}$ symmetry. Between 100 and $120 \mathrm{~cm}^{-1}$ in $B_{u}$ symmetry a powerful mode group appears, showing an energy shift of $-10 \mathrm{~cm}^{-1}$ with lower temperatures.

In conclusion, BP shows only a few mode structures, but with significant changes at the different transitions. No indications of soft modes appear but new modes appear with the unit cell doubling. In the sample with $x=0.07$ no significant changes at the phase transition were detected. Neither soft modes nor new modes or strong changes in the mode structure could be detected. Neither the modes around $2995 \mathrm{~cm}^{-1}$ $\left(B_{g}\right)$ (Fig. 3) nor those around 400 and $550 \mathrm{~cm}^{-1}\left(A_{g}\right)$ change their shapes, intensities, or split, as in pure BP. A stabilization also in the structural level is observed. The mode structure, on the other hand, is very similar to that in the pure compound in both the internal and external mode

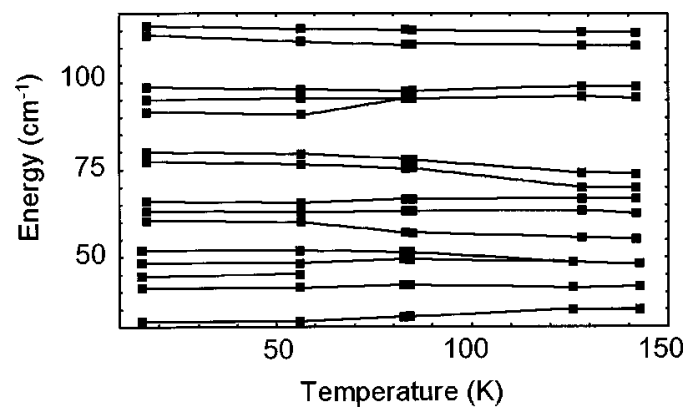

FIG. 5. Temperature dependence of some of the fitted spectra in $A_{u}$ symmetry. Solid lines are drawn to guide the eye.
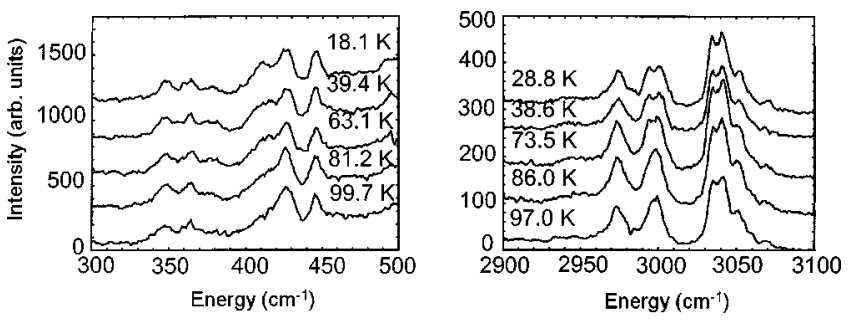

FIG. 6. Comparison of the phase behavior of the mode group around $410 \mathrm{~cm}^{-1}$ in $A_{g}$ symmetry $[X(Y Y) Z]$ for $\mathrm{BA}_{0.24} \mathrm{BP}_{0.76}$ (left), and of the mode group around $2995 \mathrm{~cm}^{-1}$ in $B_{g}$ symmetry $[Z(Y Z) X]$ for $\mathrm{BA}_{0.39} \mathrm{BP}_{0.61}$ (right).

regions, indicating a similar molecular and structural composition in both compounds. A study of the concentration dependence of the spectra will be carried out in a following section.

\section{Intermediate regions $A$ and $B$}

For both samples no major changes were detected in the Raman spectra in any of the different regions of the phase diagram. Only the regions around $\nu \sim 410$ in $A_{g}$ symmetry and, as in pure BP, the region around $2995 \mathrm{~cm}^{-1^{g}}$ in $B_{g}$ symmetry show slight changes, especially at the transition into the AF phase (Fig. 6). For $\mathrm{BA}_{0.39} \mathrm{BP}_{0.41}$ significant changes occur also around $100 \mathrm{~cm}^{-1}$ in both symmetries. Like in the previous region, the Raman spectra indicate that changes in the region of the $\nu_{2}$ and $\nu_{4}$ stretching vibrations of the tetrahedra and in the $\mathrm{C}-\mathrm{N}$ stretching vibrations are related to the dielectric behavior.

In the Raman spectra, the behavior of the $x=0.51$ compound is similar to those found in the samples in the previous region. For the other two samples within this region $(x$ $=0.62$ and 0.73 ), the changes at the phase transitions are also small, but stronger than in the other samples, especially in the rather broad modes between 300 and $500 \mathrm{~cm}^{-1}$ in both symmetries, which change in shape and strength by lowering the temperature into the AF phase.

The group of modes around $50 \mathrm{~cm}^{-1}$ in $B_{g}$ symmetry show some splittings and changes in shape and relative strength. A change in the form of the peaks at the transition into the AF phase was also observed in the region between 870 and $770 \mathrm{~cm}^{-1}$ (Fig. 7), corresponding to the stretching vibrations $\nu_{1}$ and $\nu_{2}$ of the $\mathrm{AsO}_{4}$ group, and around 890 $\mathrm{cm}^{-1}$ corresponding to a mode of the $\mathrm{PO}_{4}$ group in the pure
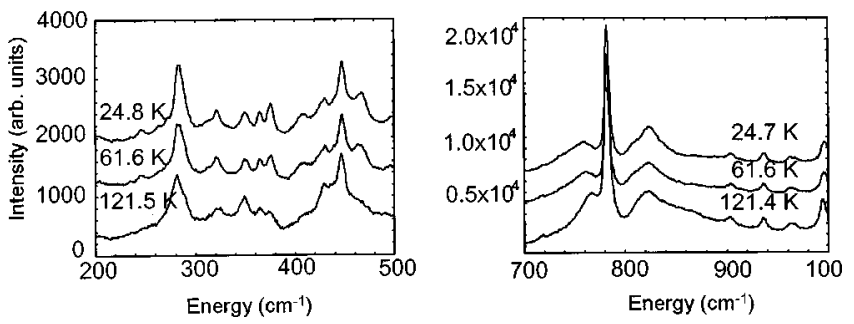

FIG. 7. Comparison of the phase behavior of the mode group between 300 and $500 \mathrm{~cm}^{-1}$ in $A_{g}$ symmetry [ $\left.Z(Y Y) X\right]$ (left) and of the mode group around $760 \mathrm{~cm}^{-1}$ in $A_{g}$ symmetry $[Z(Y Y) X]$ for $\mathrm{BA}_{0.73} \mathrm{BP}_{0.27}$ (right). 


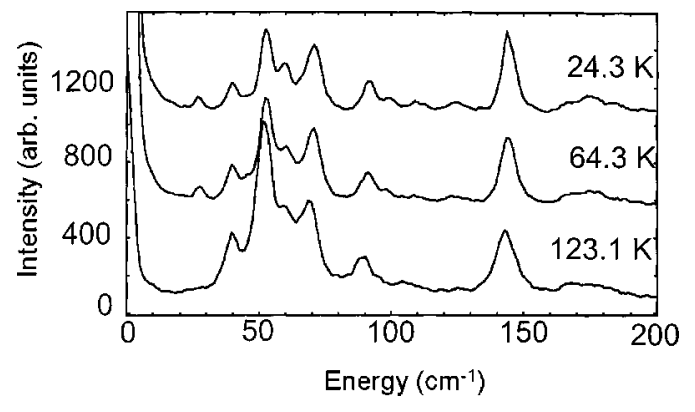

FIG. 8. Raman spectra for $x=0.62$ in $B_{g}$ symmetry [ $\left.X(Y X) Z\right]$. A new mode appears at $27.44 \mathrm{~cm}^{-1}$ with the beginning of the AF phase. The same is observed for the $x=0.73$ sample.

compounds. Slight changes were also observed in the modes around $3000 \mathrm{~cm}^{-1}$ in $B_{g}$ symmetry, but were less pronounced than in the previous region.

An important difference with respect to the other regions in the $(x, T)$ diagram is the new mode found at 27.44 and $28.38 \mathrm{~cm}^{-1}$, in $B_{g}$ symmetry, in both $x=0.62$ and 0.73 (Fig. 8 ). This mode could indicate again a doubling in the unit cell with the formation of the AF phase, such as in BP. No new mode seems to appear in the spectra taken at higher pressures, when the second anomaly in $\varepsilon^{\prime}(T)$ already exists.

The IR-reflection spectra of the two investigated crystals $x=0.51$ and 0.62 turned out to be almost equal. The $A_{u}$ spectra of both crystals were fitted with 20 oscillators. In $B_{u}$ symmetry the spectra were adapted with 21 oscillators $(x$ $=0.51)$ and with 27 oscillators $(x=0.62)$. The different number of oscillators in the $B_{u}$ spectra is not due to different mode structures, but to more noise in the spectra with the lower number.

Both compounds show marked structures only at higher wave numbers, which can be identified as internal vibrations. No indications of soft modes could be found. In $A_{u}$ symmetry the modes show no variation in the different phases. In the $B_{u}$ spectra indeed we see some modes showing anomalies in $\gamma$ and $\Delta \varepsilon$, but the anomalies could also be fluctuations with temperature (Fig. 9).
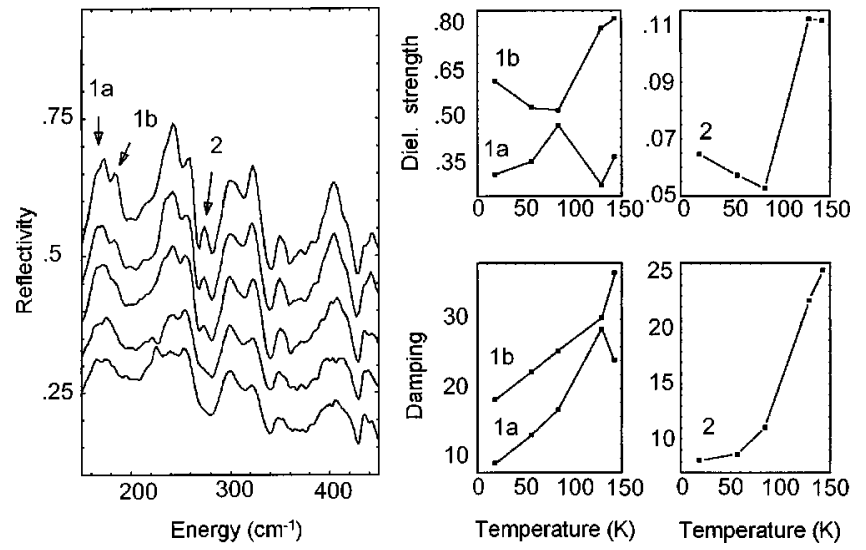

FIG. 9. Typical IR spectra of $\mathrm{BA}_{0.51} \mathrm{BP}_{49}$ (left) and fitted modes (right), $B_{u}$ symmetry. The temperatures of the spectra are, from above to below, $18.5,56.4,84.2,129.1$, and $142 \mathrm{~K}$. Solid lines (right) are drawn to guide the eye.

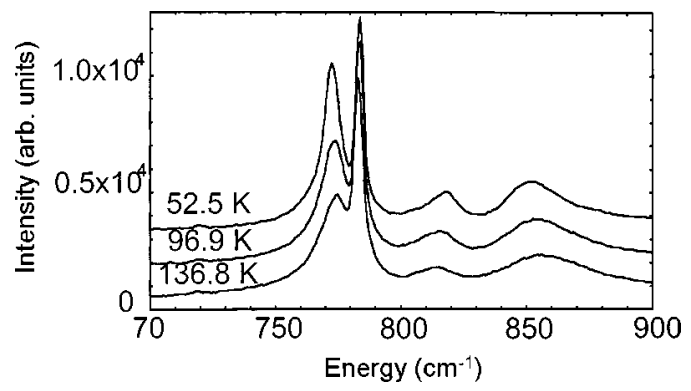

FIG. 10. Raman spectra for pure BA in $A_{g}$ symmetry $[Y(Z Z) X]$ in the region of the stretching vibrations $\left(n_{1}\right.$ and $\left.n_{3}\right)$ of the $\mathrm{AsO}_{4}$ group. Similar changes are observed in the other two samples within this region.

The Raman and the infrared results point out that, like in the regions studied before, the main changes occur in the region of the tetrahedra stretching vibrations. Therefore, they seem to be strongly related to the dielectric behavior of the different phase. Despite the significant changes occurring in the dielectric response of the material under hydrostatic pressure, ${ }^{1-4}$ the Raman spectra taken under pressure do not present major differences with respect to those taken at normal pressure.

\section{BA region}

Comparing the Raman spectra taken in the different regions of the phase diagrams reveals no major differences for BA and no indication of a soft mode has been found. ${ }^{22}$ The small structural changes are localized at $456.8 \mathrm{~cm}^{-1}\left(A_{g}\right)$ where the mode changes its form and increases its intensity strongly with lower temperatures. This group of modes can be identified with the $\nu_{2}=349$ and $\nu_{4}=463$ vibrations of the $\mathrm{AsO}_{4}$ tetrahedra. The most important changes are localized in the region between 770 and $870 \mathrm{~cm}^{-1}$ in both symmetries, assigned to the stretching vibrations $\left(\nu_{1}\right.$ and $\nu_{3}$ ) of the $\mathrm{AsO}_{4}$ group (Fig. 10). These changes are continuous, i.e., they are not restricted to the transition points. Only at the transition into the FE phase does a new mode appear around $850 \mathrm{~cm}^{-1}$ (Fig. 11). Slight changes in the region around $270 \mathrm{~cm}^{-1}\left(A_{g}\right)$ have also been observed.

In the IR-reflection spectra of BA, 29 and 25 modes were fitted in the $A_{u}$ and the $B_{u}$ spectra, respectively. In general a clear energy shift of the modes could not be observed. In $B_{u}$

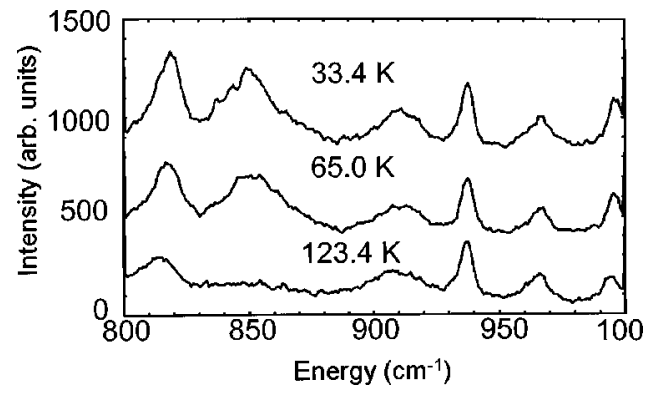

FIG. 11. Raman spectra for $x=0.86$ in $B_{g}$ symmetry [ $\left.Y(Z Y) X\right]$. A new mode appears around $850 \mathrm{~cm}^{-1}$. Similar changes are observed in the other two samples within this region. 


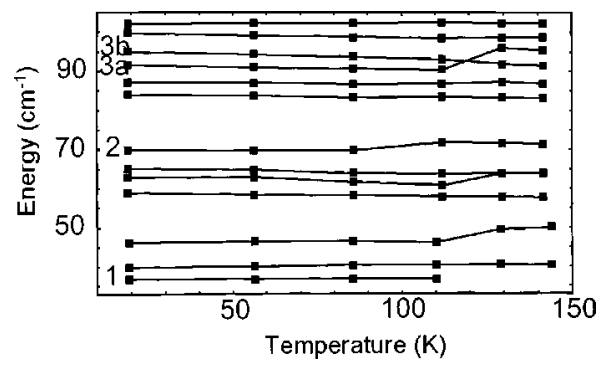

FIG. 12. Temperature dependence of some of the fitted spectra in $B_{u}$ symmetry. Solid lines are drawn to guide the eyes.

symmetry a new mode appears at $36.8 \mathrm{~cm}^{-1}$ with the beginning of the FE phase (Fig. 12). Anomalies of $\gamma$ and $\Delta \varepsilon$ in both symmetries appear frequently between 85 and $110 \mathrm{~K}$, consequently, at temperatures not connected with the transitions but with the second contribution to the polarization (Fig. 13).

$A_{u}$ spectra show no significant anomalies in $\gamma$ and $\Delta \varepsilon$. Between 106 and $111 \mathrm{~cm}^{-1}$ a new mode appears at temperatures $T<85.2 \mathrm{~K}$. Only the mode at $118 \mathrm{~cm}^{-1}$ shows an energy shift of $-9.1 \mathrm{~cm}^{-1}$ stepping in the FE phase (Fig. 13).

In conclusion the mode structures of BA are more abundant than in BP, but less phase-specific features can be observed. No indications of a soft mode were found. It is conspicuous that mode changes (new modes, anomalies in $\gamma$ and $\Delta \varepsilon$ ) appear between 85 and $120 \mathrm{~K}$. Obviously structural changes are not restricted to the transition temperatures $T_{c}$, but take place over a large region below $T_{c 2}$.

The Raman spectra of both the $x=0.86$ and 0.94 samples are similar to those obtained for pure BA: small changes have been observed around $270 \mathrm{~cm}^{-1}$ in $A_{g}$ symmetry and in the group of modes around $450 \mathrm{~cm}^{-1}$, as in the internal region $\mathrm{B}$, but are much smaller in both symmetries (strong increase of the intensity down to the lower temperatures). Like in the other samples within this region the most conspicuous changes occur in the region of the internal modes where, together with some small changes in the region around $2995 \mathrm{~cm}^{-1}$ in $B_{g}$ symmetry, a new mode appears at $850 \mathrm{~cm}^{-1}$ in the FE phase and increases down to the lowest-
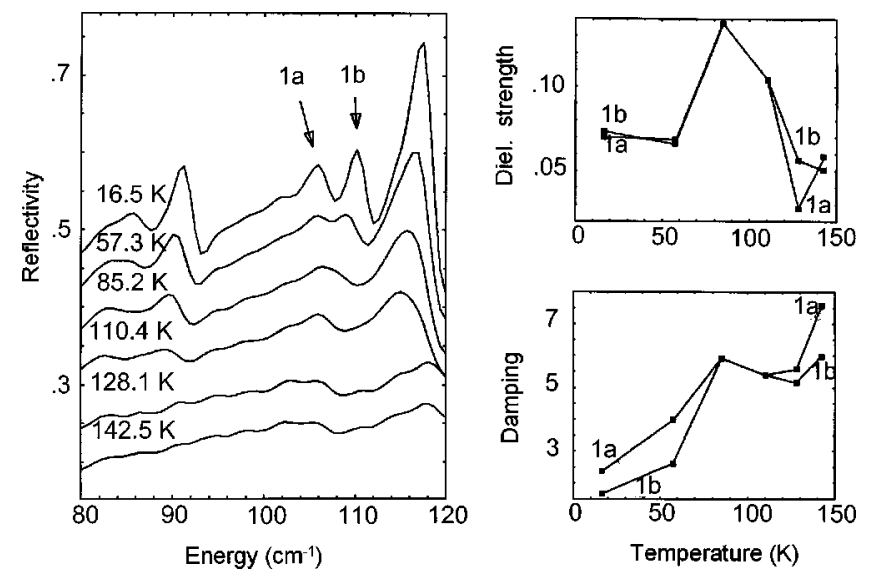

FIG. 13. Typical IR spectra of BA (left) and fitted modes (right), $A_{u}$ symmetry. Solid lines (right) are drawn to guide the eyes.
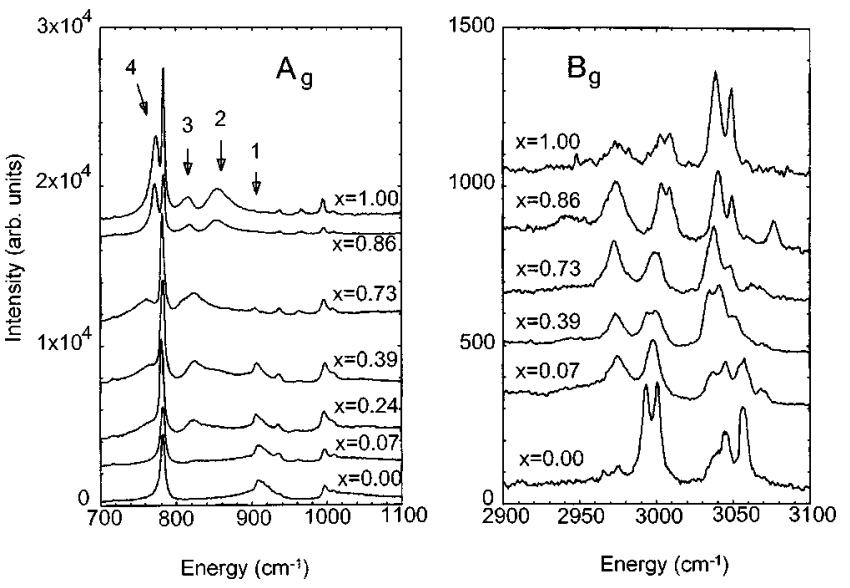

FIG. 14. Raman spectra of different concentrations in the ordered phase $(T \sim 60 \mathrm{~K})$ in the region of the internal modes. Left: $A_{g}$ symmetry. Right: $B_{g}$ symmetry.

temperature phase (Fig. 11). Also the modes around 780 $\mathrm{cm}^{-1}$ demonstrate the changes described in Fig. 10.

The mode structure and the changes in it are also similar in the whole FE region of the $(x, T)$ phase diagram in both the external and internal mode regions and they present some evidence of very small structural changes, i.e., a similar structural and molecular coordination between the different concentrations. As in the previous regions, no major differences are detected in the spectra taken under hydrostatic pressure.

The Raman and IR experiments show that small structural changes occur in the whole temperature range beginning with the transition into the FE phase; they are not restricted to the transition points. These changes are especially strong in the region of the stretching vibrations of the $\mathrm{AsO}_{4}$ group and seem to be responsible for the dielectric behavior.

\section{B. Concentration dependence}

In the present section we will study the concentration dependence of the spectra in order to characterize the structural changes that accompany the observed features of the phase behavior. We will concentrate the study on the Raman spectra, since more samples were measured by this technique, at low temperature, the features of the spectra are clearer, and the variations with temperature, as already pointed out, do not modify the spectra substantially.

The comparison of the Raman spectra in the different concentrations demonstrates their strong similarity in both the internal and external mode regions. New mode groups do not appear in the mixed crystals. As a consequence, the molecular structure and the molecular arrangement in the crystal must be similar in both the mixed crystals and the pure compounds: the quasi-1D structure must be preserved. The most striking feature in this comparison occurs in the group of modes between 700 and $900 \mathrm{~cm}^{-1}$ (Fig. 14). Together with other minor variations, the strong change in the mode structure in this region [two modes disappear (1 and 2) and other decreases abruptly in intensity (4)] indicates the transition in concentration from a FE to an AF ordered phase. Moreover, 

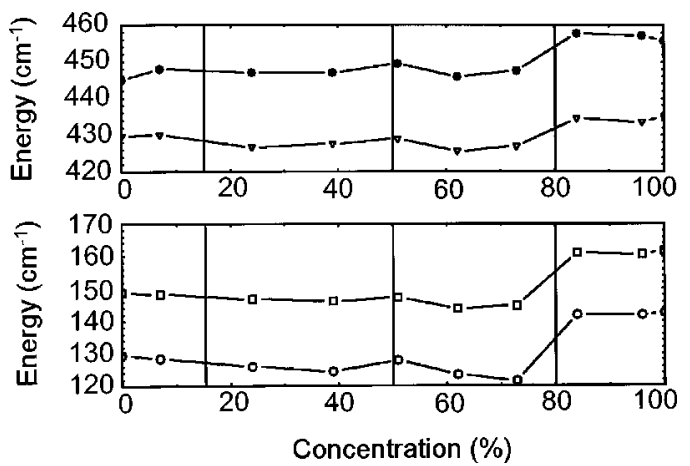

FIG. 15. Concentration dependence of the modes around 130 and $430 \mathrm{~cm}^{-1}$ in $A_{g}$ symmetry. Solid lines are drawn to guide the eyes.

a further change in this region [one mode disappears (3)] indicates the transition from the intermediate to the BP region. Other changes in the mode structure occur more or less continuously and others, such as the modes around 130, 150, 430 , and $445 \mathrm{~cm}^{-1}$ (Fig. 15), show an abrupt shift to higher frequencies at the transition in concentration from the AF to the FE phase.

From this data one can conclude that the FE or AF character of the ordered phase is connected with the tetrahedral degrees of freedom. In the same way, the tetrahedra seem to determine the kind and components of the phase transitions for each concentration. The latter seems to occur together with a participation of the betaine molecule, especially of the methyl and buthyl groups as already pointed out in Ref. 23.

The possibility of phase segregation was considered in order to explain the complex features of the dielectric constant of these compounds, especially in the regions in which mixed phases seem to exist. In this case the Raman and infrared spectra for a given concentration in a given phase could be simulated by a superposition of the spectra of the pure compounds at a corresponding temperature or phase.

We tried to simulate the spectra to the function [SIM] $=\left[r^{*}[\mathrm{BA}]+(1-r)^{*}[\mathrm{BP}]\right]$, where SIM stands for the simulated spectra, BA and BP for the corresponding spectra of the pure compounds, and $r$ represents a free parameter that was changed starting from the initial composition of the mixed crystal in order to get the best fits. Though reasonable fitting could be achieved for some concentrations and/or spectral regions, the general results point out that the spectra of the mixed compounds show features (in the number, the energy, and the intensity of the modes) that cannot be explained by a simple superposition of the spectra of the pure compounds.

\section{DISCUSSION AND CONCLUSION}

The Raman and IR spectra do not exhibit strong changes or shifts at the different transitions between the regions of the phase diagram. ${ }^{1-4}$ Examples are shown in Fig. 14 for internal modes of the tetrahedra and the $\mathrm{C}-\mathrm{H}$ stretching vibrations. From this finding the conclusion can be drawn that either the system of the polarization dipoles and the molecular structures are rather uncoupled with each other, or that rather small structural changes have a large influence on the dipolar order. The observed changes are usually found in the regions around 450 and $700-900 \mathrm{~cm}^{-1}$ and in the region around $3000 \mathrm{~cm}^{-1}$. On the other hand it is evident, after the continuous behavior of the phase-transition point with concentration, that a relation between the concentration and the transition temperature exists [especially in the anomalies in $\left.\varepsilon^{\prime}(T)\right]$.

The comparison of the spectra at the different concentrations demonstrates that the molecular structure and the molecular arrangement in the crystal must be similar in both the mixed crystals and the pure compounds. Strong changes in the mode structure in the region of the internal modes of the $\mathrm{PO}_{4}$ and $\mathrm{AsO}_{4}$ tetrahedra (Fig. 14), together with other minor variations, indicate the transition in concentration from the FE to the AF ordered phase. Moreover, a further change in this wave-number region indicates the transition from the intermediate to the $\mathrm{BP}$ region.

It has been shown in deuterated BP using ${ }^{2} \mathrm{H}$ NMR (Refs. 24 and 25) that the phase transition $\mathrm{PE} \leftrightarrow \mathrm{AF}$ exhibits an "order-disorder" -like behavior where in the ordered phase the molecules (tetrahedra, betaine) occupy one of two positions tilted with respect to the $m_{y}$ mirror plane. This tilting is triggered by the protons or deuterons, respectively, in the $\mathrm{H}$ bonds. ${ }^{26}$ Other changes in the mode structure occur more or less continuously and again others, such as the external modes around 130 and $150 \mathrm{~cm}^{-1}$ and the modes at 430 and $445 \mathrm{~cm}^{-1}$ (Fig. 15), display an abrupt shift to higher frequencies at the transition in concentration from the AF to the FE phase. From these data one can conclude that the FE or AF character of the ordered phase is connected with the degrees of freedom of the tetrahedra. In the same way these units determine the type and the modes triggering the phase transitions for each concentration. The latter seems to occur together with a participation of the betaine molecule, especially of the methyl and buthyl groups as already pointed out in Ref. 23.

In the BA-BP system proton tunneling might play a certain role, since the lengths of the $\mathrm{H}$ bonds in the pure compounds are found close to $2.5 \AA$, where a symmetrical double-minimum potential can be expected. There is, however, no clear experimental indication for such an effect.

There is some evidence for the important role of the proton degrees of freedom in $\mathrm{BA}, \mathrm{BP},{ }^{27}$ and, especially, in BPI and the $\operatorname{BP}: \operatorname{BPI}(x)$ system. $^{24}$ The distortion of the tetrahedra $^{28,29}$ was shown to contribute to the polarization. This distortion was also proposed as the mechanism responsible for the ordered phase, whereas only a partial proton order was found. Our experiments, especially the Raman investigations, support the importance of the tetrahedra dynamic in the various phases both with temperature and concentration. The experiments under hydrostatic pressure and the fits with the quasi-1D Ising model give evidence for the importance of the interactions in the plane perpendicular to the polar axis. ${ }^{1,2}$ The striking differences with the BP/BPI system $^{11,12}$ can also be explained by making the tetrahedra and the bonds with the betaine molecule responsible for the dipolar behavior. These are the main differences between the two mixed systems and also between the three pure compounds. 
From our experimental results ${ }^{1-4}$ we propose that the local freezing of the protons in their double-well potential should occur at lower temperatures than the transition into the ordered phase, it is independent of the dipoles formed at these transitions, and it corresponds to the low-temperature relaxation process fitted with an Arrhenius law. On the other hand, the freezing process fitted with a Vogel-Fulcher formula is obviously related with the dipolar structure originated at the transition into the ordered phase, which can be associated with the tetrahedra degrees of freedom. ${ }^{1,2}$
${ }^{1}$ S. Lanceros-Méndez, G. Schaack, and A. Klöpperpieper, Frontiers in Science and Technology-Science and Technology of Ferroelectrics, Ferroelectrics, Vol. 2 (Stefan University, La Jolla, California, 2001).

${ }^{2}$ S. Lanceros-Méndez and G. Schaack, Ferroelectrics 226, 107 (1999).

${ }^{3}$ S. Lanceros-Méndez and G. Schaack, J. Korean Phys. Soc. 32, S850 (1998).

${ }^{4}$ S. Lanceros-Méndez, M. Manger, G. Schaack, and A. Klöpperpieper, Ferroelectrics 184, 281 (1995); S. Lanceros-Méndez, G. Schaack, M. Köhler, and A. Klöpperpieper, ibid. 176, 73 (1996); S. Lanceros-Méndez, Ph.D. thesis, Universität Würzburg, 1996. ${ }^{5}$ M. Maeda, Ferroelectrics 96, 269 (1989).

${ }^{6}$ M. Maeda and I. Suzuki, Ferroelectrics 108, 351 (1990).

${ }^{7}$ S. Hayase, T. Koshiba, H. Terauchi, M. Maeda, and I. Suzuki, Ferroelectrics 96, 221 (1989).

${ }^{8}$ Y-H. Kim, B-G. Kim, J-J. Kim, T. Mochida, and S. Miyajima, J. Phys.: Condens. Matter 8, 6095 (1996).

${ }^{9}$ T. Yoshida, H. Mashiyama, and T. Mochida, J. Korean Phys. Soc. 35, 1409 (1999).

${ }^{10}$ T. Yoshida, H. Mashiyama, and T. Mochida, J. Phys. Soc. Jpn. 70, 1598 (2001).

${ }^{11}$ H. Ries, R. Böhmer, I. Fehst, and A. Loidl, Z. Phys. B: Condens. Matter 99, 401 (1996).

${ }^{12}$ M. L. Santos, M. R. Chaves, A. Almeida, A. Klöpperpieper, H. E. Müser, and J. Albers, Ferroelectr. Lett. Sect. 15, 17 (1993).

${ }^{13}$ E. Courtens, Ferroelectrics 72, 229 (1987).

${ }^{14}$ W. Schildkamp and J. Spilker, Z. Kristallogr. 168, 159 (1984).

${ }^{15}$ W. Schildkamp, J. Spilker, and G. Schäfer, Z. Kristallogr. 168, 187 (1984).
${ }^{16}$ T. Yoshida, H. Mashiyama, and T. Mochida, J. Phys. Soc. Jpn. 70, 569 (2001)

${ }^{17}$ H. J. Rother, J. Albers, A. Klöpperpieper, and H. E. Müser, Jpn. J. Appl. Phys., Suppl. 24, 384 (1985).

${ }^{18}$ M. Born and K. Huang, Dynamical Theory of Crystal Lattices (Clarendon, Oxford, 1954).

${ }^{19}$ O. Freitag, H. J. Bruckner, and H. G. Unruh, Z. Phys. B: Condens. Matter 61, 75 (1985).

${ }^{20}$ K. Hara, H. Umeda, Y. Ishibashi, and I. Suzuki, J. Phys. Soc. Jpn. 58, 4215 (1989).

${ }^{21}$ M. L. Santos, A. Almeida, M. R. Chaves, A. Klopperpieper, J. Albers, J. A. Gomes-Moreira, and F. Gervais, J. Phys.: Condens. Matter 9, 8119 (1997).

${ }^{22}$ J. Agostinho-Moreira, A. Almeida, M. R. Chaves, M. F. Mota, A. Klopperpieper, and Filipa Pinto, J. Phys.: Condens. Matter 10, 6825 (1998).

${ }^{23}$ H. Ohki, N. Nakamura, and H. Chihara, Ferroelectr. Lett. Sect. 8, 19 (1987).

${ }^{24}$ H. Bauch, Ph.D. thesis, Universität Leipzig, 1994; H. Bauch, R. Böttcher, and G. Völkl, Phys. Status Solidi B 178, K39 (1993); 179, K41 (1993).

${ }^{25}$ P. Freude and D. Michel, Phys. Status Solidi B 195, 297 (1996).

${ }^{26}$ P. Freude, D. Michel, J. Totz, and A. Klöpperpieper, Ferroelectrics 208, 93 (1998).

${ }^{27}$ S. Launer, M. le Maire, G. Schaack, and S. Haussühl, Ferroelectrics 135, 257 (1992).

${ }^{28}$ G. Fischer, H. J. Brückner, A. Klöpperpieper, H. G. Unruh, and A. Levstik, Z. Phys. B: Condens. Matter 79, 301 (1990).

${ }^{29}$ K. H. Ehses and J. Spilker, Ferroelektrizität 89, edited by G. Schmitt and A. Rost (Wissenschaftliche Beiträge Martin Luther Universität, Halle, 1990). 